\title{
Mucinous tubular and spindle cell carcinoma of the kidney
}

\author{
Yuichiro Suzuki • Takuya Koie • Hayato Yamamoto • \\ Shingo Hatakeyama - Takahiro Yoneyama • Yasuhiro Hashimoto • \\ Noritaka Kamimura $\cdot$ Chikara Ohyama
}

Received: 8 February 2012/ Accepted: 15 March 2012/Published online: 11 April 2012

(C) The Japan Society of Clinical Oncology 2012

\begin{abstract}
Mucinous tubular and spindle cell carcinoma (MTSCC) is a low-grade renal epithelial neoplasm that has recently been recognized as a specific entity in the 2004 World Health Organization classification of renal cell carcinoma. A 69-year-old woman was referred to our hospital because of incidental left renal tumor. Abdominal computed tomography showed a hypovascular tumor in the left kidney. Left radical nephrectomy was performed. Hematoxylin and eosin staining revealed cuboidal and oval or spindle-shaped cells in tubular and papillary patterns embedded in the myxoid stroma. Immunohistochemically, the tumor cells were positive for alpha-methylacyl-CoA racemase. On the basis of these findings, we diagnosed the patient as having MTSCC.
\end{abstract}

Keywords Mucinous tubular and spindle cell carcinoma $\cdot$ Renal cell carcinoma $\cdot$ Papillary renal cell carcinoma

\section{Introduction}

Mucinous tubular and spindle cell carcinoma (MTSCC) has recently been classified in the 2004 World Health Organization classification of renal tumors [1]. It is a relatively lowgrade carcinoma and is associated with a good prognosis [2]. The clinical characteristics of MTSCC are similar to those of papillary renal cell carcinoma (RCC) with sarcomatoid transformation. Hence, a precise differential diagnosis

Y. Suzuki · T. Koie $\cdot$ H. Yamamoto $\cdot$ S. Hatakeyama

T. Yoneyama $\cdot$ Y. Hashimoto $\cdot$ N. Kamimura $\cdot$ C. Ohyama $(\bowtie)$

Department of Urology, Hirosaki University, Graduate School

of Medicine, 5 Zaifucho, Hirosaki 036-8562, Japan

e-mail: coyama@cc.hirosaki-u.ac.jp between both of these diseases is important because they have different biological behaviors [3]. Herein, we report a case of MTSCC and review the relevant literature.

\section{Case report}

A 69-year-old woman visited our hospital with a chief concern of a left renal tumor, which was incidentally identified by a screening computed tomography (CT) for bilateral femoral vein thrombosis. A metal stent was indwelled into the inferior vena cava. Laboratory evaluations revealed anemia $(9.4 \mathrm{~g} / \mathrm{dl}$ ). CT revealed a hypovascular tumor (size $4.3 \times 3.5 \times 4.6 \mathrm{~cm}$ ) in the left kidney (Fig. 1a, b). Left radical nephrectomy was performed. Macroscopic examination revealed a solid, well-demarcated, yellowish-white tumor, measuring $4.3 \times 4 \mathrm{~cm}$ in size, in the lower pole of the resected kidney (Fig. 2). Histological examination of the tumor revealed cuboidal and oval or spindle-shaped cells arranged in tubular and papillary patterns embedded in the myxoid stroma (Fig. 3). Tumor cells invaded into the left renal vein. Immunohistochemically, the tumor cells were strongly positive for alpha-methylacyl-CoA racemase (AMACR), CAM5.2, and P504S (Fig. 4a, b, c), and negative for vimentin, smooth muscle actin, CD10, and molecular weight cytokeratin. On the basis of these findings, we diagnosed the patient as having MTSCC, stage pT3bNOM0. The patient was asymptomatic and free of the disease 3 years after the diagnosis.

\section{Discussion}

MTSCC is characterized by tightly packed tubules separated by pale mucinous stroma and a spindle cell 

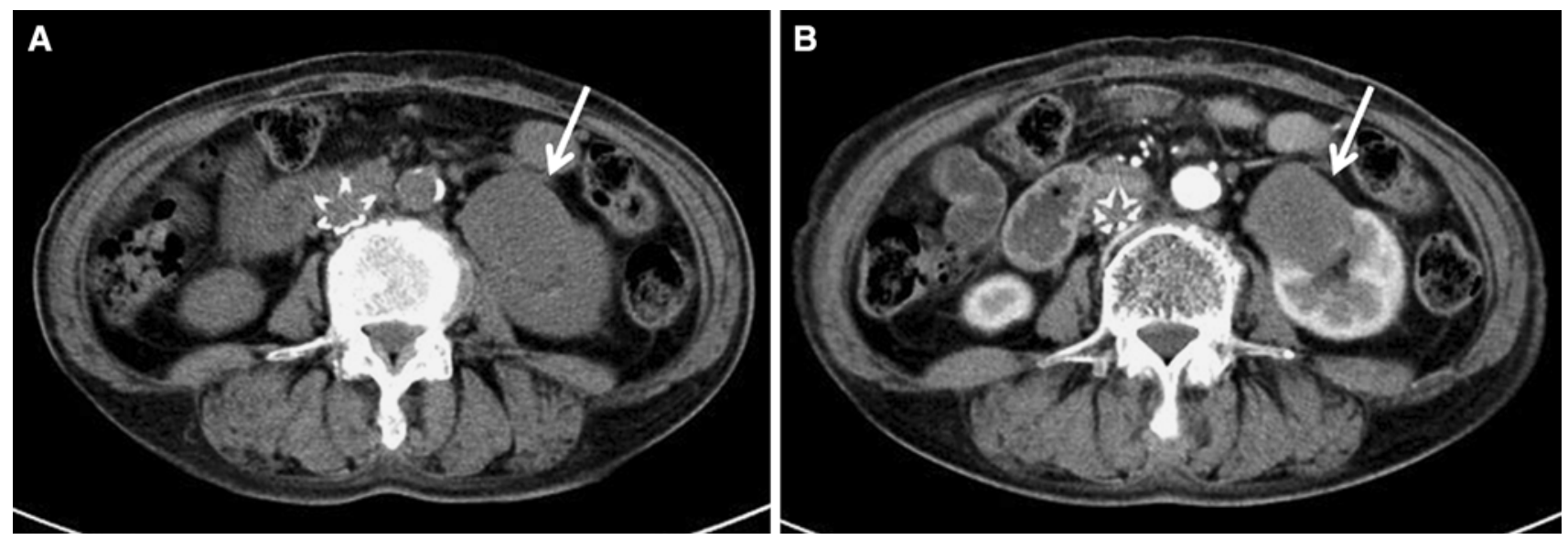

Fig. 1 Abdominal computed tomography revealed a hypovascular tumor $(4.3 \times 3.5 \times 4.6 \mathrm{~cm})$ (arrow) in the lower pole of the left kidney (a plain, b enhanced)

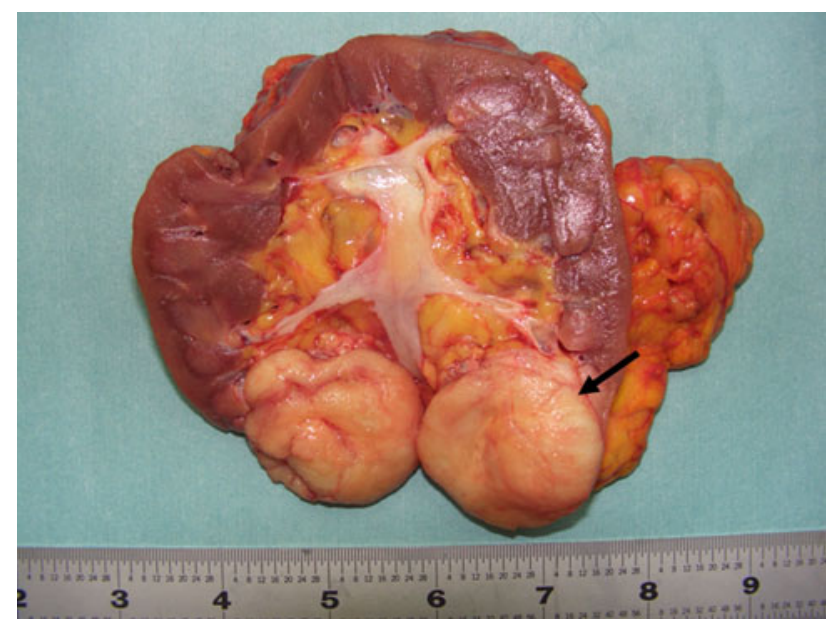

Fig. 2 Macroscopic findings of the resected kidney revealed a welldemarcated tumor in the lower portion. The tumor had a yellowishwhite cut surface (arrow)

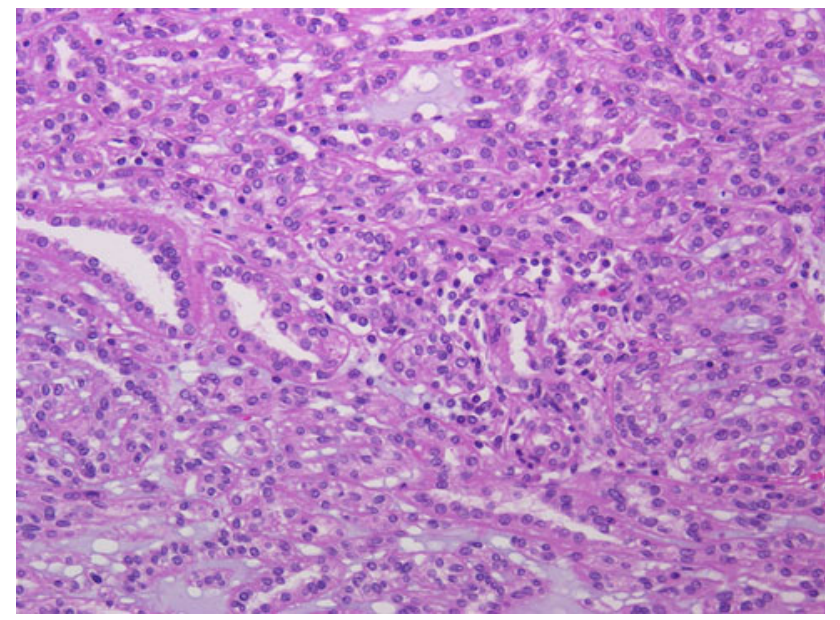

Fig. 3 The tumor consisted of cuboidal and oval or spindle-shaped cells arranged in tubular and papillary patterns embedded in the myxoid stroma $(H \& E$, reduced from $\times 200)$ component [1]. Renal tumors with these histologic features were previously categorized as a variant of solid papillary RCC with compressed and elongated papillae, metanephric adenomas, or unclassified or sarcomatoid carcinomas [4]. Sarcomatoid transformation is the most important feature for the differential diagnosis of MTSCC from papillary RCC [3]. Papillary RCC has an overall 5-year survival rate of approximately $86 \%$ [5]. Sarcomatoid or spindle cell differentiation is reported to occur in 3-5\% of papillary RCC cases and is considered as a strong indicator for adverse prognostic outcome with 1- and 5-year survival rates of 59 and $22 \%$, respectively [6]. In addition, unlike MTSCCs that rarely metastasize, papillary RCCs, the second most common RCC subtype (occurrence 11-18\%), have been shown to metastasize to the lymph nodes in $7 \%$ of the cases [5]. Thus, accurately distinguishing MTSCC from papillary RCC with sarcomatoid transformation is absolutely essential [5].

MTSCCs have been reported to have no characteristics on CT and magnetic resonance imaging (MRI) [7, 8]. Thus, the preoperative differential diagnosis of MTSCC from papillary RCC may be difficult on the basis of either CT or MRI.

MTSCCs are polymorphic neoplasms showing a wide range of morphologic variability [2]. Histologically, they are generally characterized by the presence of cuboidal and spindle cells [9]. However, there are several morphological variants of MTSCC that are difficult to diagnose. Some cases might show predominance of various components: some MTSCCs may show spindle cell predominance and mimic sarcomatoid carcinomas, and others may show tubular cell predominance and mimic the solid variant of papillary RCC [2]. Other characteristic features include tumors with poor mucin content, areas with extracellular hyaline matrix and blue-tinged mucin within tubules, and solid areas with epithelioid cells [2]. 

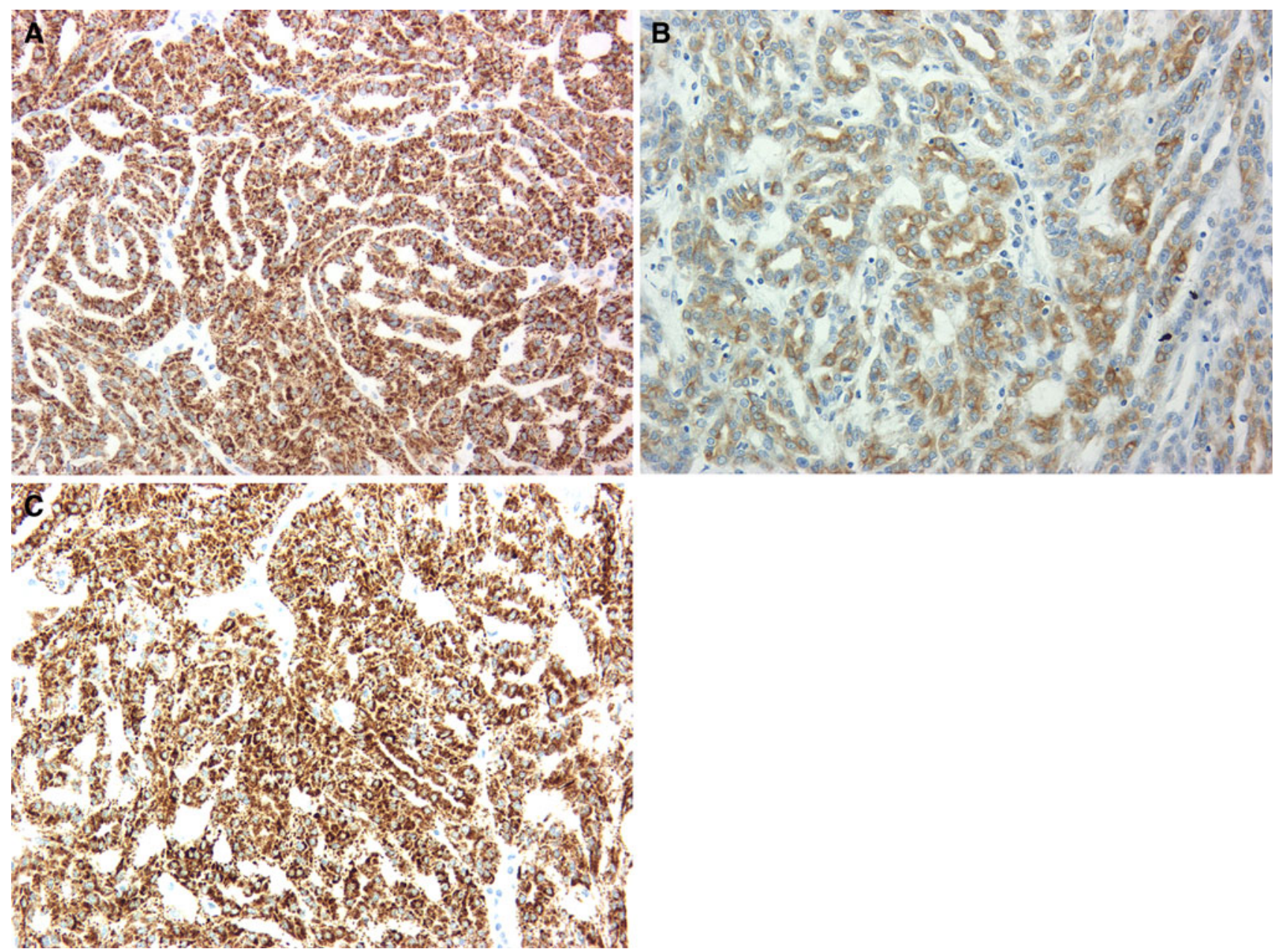

Fig. 4 Immunohistochemistry showed that the tumor cells were positive for alpha-methylacyl-CoA racemase (a), CAM5.2 (b), and P504S (c) (reduced from $\times 200$ )

MTSCCs are known to originate from the collecting duct epithelium or cells of the loop of Henle [8]. Tumor cells of MTSCCs have a complex and variable immunophenotype [10]. Many MTSCC tumors are positive for AMACR, epithelial membrane antigen, and cytokeratin $\mathrm{AE} 1 / 3$ [11]. CD10 immunoreactivity is noted in $93-100 \%$ of papillary RCCs [9] and in only $15 \%$ of MTSCCs [10].

Papillary RCC is characterized by chromosomal gains, particularly chromosomes 7 and 17, and the loss of chromosome Y [12]. On the other hand, MTSCCs are characterized by the combination of losses of chromosomes $1,4,6,8,13$, and 14 and gains of chromosomes 7, 11, 16, and 17 [1]. These chromosomal abnormalities are potentially useful for the early and accurate distinction of the different histotypes of RCCs.

MTSCCs and papillary RCCs have a similar immunohistochemical staining pattern as well as histological characteristics; this may hinder the differential diagnosis. In the absence of any significant cell abnormalities, pathologists and urologists should be aware of the histological spectrum of MTSCCs to ensure an accurate diagnosis.
Conflict of interest The authors have no conflict of interest.

\section{References}

1. Lopez-Beltran A, Scarpelli M, Montironi R et al (2006) 2004 WHO classification of the renal tumors of the adults. Eur Urol 49:798-805

2. Gaafar A, Valenti C, Echevarria C et al (2006) Renal mucinous and tubular spindle cell carcinoma: a clinicopathological study of 4 cases. Ann Saudi Med 26:466-470

3. Cossu-Rocca P, Eble JN, Delahunt B et al (2006) Renal mucinous tubular and spindle carcinoma lacks the gains of chromosomes 7 and 17 and losses of chromosome $\mathrm{Y}$ that are prevalent in papillary renal cell carcinoma. Mod Pathol 19:488-493

4. Ferlicot S, Lupo A, Lazure T et al (2005) A novel entity: lowgrade mucinous tubular renal carcinoma. Histopathology 47: 218-219

5. Cheville JC, Lohse CM, Zincke H et al (2003) Comparison of outcome and prognostic features among histologic subtypes of renal cell carcinoma. Am J Surg Pathol 27:612-614

6. Avery AK, Becksted J, Renshaw AA et al (2000) Use of antibodies to RCC and CD10 in the differential diagnosis of renal neoplasms. Am J Surg Pathol 24:202-210 
7. Hara N, Kawaguchi M, Koike H et al (2004) Low-grade renal epithelial tumor originating from the distal nephron. Int J Urol 11:68-73

8. Otani M, Shimizu T, Serizawa H et al (2001) Low-grade renal cell carcinoma arising from the lower nephron: a case report with immunohistochemical, histochemical and ultrastructural studies. Pathol Int 51:954-960

9. Razoky C, Schmahl CE, Bogner S et al (2002) Low-grade tubular mucinous renal neoplasms: Morphologic, immunohistochemical, and genetic features. Mod Pathol 15:1162-1171

10. Paner GP, Srigley JR, Radhakrishnan A et al (2006) Immunohistochemical analysis of mucinous tubular and spindle cell carcinoma and papillary renal cell carcinoma of the kidney: significant immunophenotypic overlap warrants diagnostic caution. Am J Surg Pathol 30:13-19

11. Parwani AV, Husain AN, Epstein JI et al (2001) Low-grade myxoid renal epithelial neoplasms with distal nephron differentiation. Hum Pathol 32:506-512

12. Jiang F, Richter J, Schraml P et al (1998) Chromosomal imbalances in papillary renal cell carcinoma: genetic differences between histological subtypes. Am J Pathol 153:1467-1473 